RESULTS: 304 patients enrolled in the extension study. At Week 54, the mean (standard error) change in AIMS score was -5.1 (0.52). After 6 weeks of deutetrabenazine treatment, the proportion of patients who achieved treatment success was $58 \%$ per the CGIC and $53 \%$ per the PGIC, and by Week 54 was $72 \%$ per the CGIC and $59 \%$ per the PGIC, thus demonstrating maintenance or enhancement of benefit over time. Deutetrabenazine was well tolerated for up to 54 weeks, and compared with the ARM-TD and AIM-TD studies, no new safety signals were detected.

CONCLUSIONS: 54 weeks of deutetrabenazine treatment was generally efficacious, safe, and well tolerated in patients with TD.

Presented at: The American Psychiatric Association 2017 Annual Meeting; May 20-24, 2017; San Diego, California, USA.

FUNDING ACKNOWLEDGEMENTS: This study was funded by Teva Pharmaceutical Industries, Petach Tikva, Israel.

150

\section{Estimation of an MCID for AIMS Total Score Change in Tardive Dyskinesia}

Martha Sajatovic, $\mathrm{MD}^{\prime}$; Andrew J. Cutler, $\mathrm{MD}^{2}$; Khodayar Farahmand, PharmD ${ }^{3}$; Joshua Burke, $M S^{3}$; Scott Siegert, PharmD ${ }^{4}$; and Grace S. Liang, $M D^{5}$

${ }^{1}$ Neurological and Behavioral Outcomes Center, University Hospitals Cleveland Medical Center, Cleveland, $\mathrm{OH}$

${ }^{2}$ Meridien Research, Tampa, FL

${ }^{3}$ Director, Head of Medical Communications, Neurocrine Biosciences, Inc., San Diego, CA

${ }^{4}$ Executive Director, Head of Medical Affairs, Neurocrine Biosciences, Inc., San Diego, CA

${ }^{5}$ Medical Director, Clinical, Neurocrine Biosciences, Inc., San Diego, CA

ABSTRACT: Background: The efficacy of valbenazine (INGREZZA) in tardive dyskinesia (TD) was demonstrated in placebo-controlled clinical trials, based on the Abnormal Involuntary Movement Scale (AIMS) total score (sum of items 1-7). In these trials, mean changes in the AIMS total score were significantly greater with valbenazine $80 \mathrm{mg}$ than with placebo. Currently, no minimal clinically important difference (MCID) has been established for the AIMS total score in patients with TD. Using valbenazine trial data, analyses were conducted to establish a MCID for AIMS total score in TD.

METHODS: Data were pooled from three 6-week trials: KINECT (NCT01688037), KINECT 2 (NCT01733121), KINECT 3 (NCT02274558). Using the Clinical Global
Impression ofChange (CGI-TD) as an anchor comparison, AIMS total score changes from baseline to Week 6 were summarized for all study participants (pooled valbenazine and placebo groups) with a "minimal" CGITD score of $\leq 3$ (minimally improved or better) or "robust" $\leq 2$ (much improved or better) at Week 6 .

RESULTS: In the pooled population ( $\mathrm{N}=373), 72 \%$ and $29 \%$ of all participants had CGI-TD scores of $\leq 3$ and $\leq 2$, respectively. The median (maximum, minimum) change from baseline in AIMS total score at Week 6 was -2 $(-13,8)$ in participants with CGI-TD score $\leq 3$ and -3 $(13,8)$ in participants with a score $\leq 2$.

CONCLUSION: Pooled data from 3 randomized, doubleblind, placebo-controlled trials suggest that a 2 point decrease in AIMS total score may represent the minimal clinically meaningful improvement. Larger AIMS score improvements were associated with "much improved" or "very much improved" CGI TD assessments.

FUNDING ACKNOWLEDGEMENTS: This study was funded by Neurocrine Biosciences, Inc.

\section{1}

\section{Improving the Systematic Use of Pharmacogenetic Testing for Depression Prescribing \\ Lauren Thomann Hughes, DNP, ARNP, PMHNP-BC}

Psychiatric/Mental Health Nurse Practitioner, Abbe Center for Community Mental Health, Iowa City, IA

ABSTRACT: Study Objectives: The purpose of this project was to systematize the use of pharmacogenetic testing (PGT) among psychiatric prescribers. The use of PGT in clinical practice is inconsistent despite the evidence supporting its efficacy (Burke, Love, Jones, \& Fife, 2016). The question to be answered is: In patients with major depressive disorder (MDD), how is PGT currently used in clinical practice compared to use after implementation of practice change interventions?

METHOD: This study was conducted among 4 psychiatric prescribers in a behavioral health clinic. 3 interventions were utilized to change practice. An educational inservice was delivered to address the PGT knowledge gap. A protocol for identifying patients that may benefit from PGT was developed, indicating PGT was warranted for patients with non-remitting moderate to severe MDD and at least 2 medication failures from 2 different classes. Next, a medication failure documentation template and the PGT report were integrated into the EHR. A baseline survey was administered before the in-service, assessing 
prescriber PGT perceptions and current parameters and barriers for use. Follow-up surveys were administered 3 months post-implementation. Project processes were measured by assessing the rate of medication failure template usage, as well as thePGT EHR upload rate.

RESULTS: A comparison of baseline and follow-up surveys indicated there was little change in prescriber view of test utility, receptiveness, and likelihood of use. This may be attributed to previous experience with testing and to PGT manufacturer education. View of parameters and barriers for use did change. Key parameter for use changes included patient experience of adverse reaction (increase) and only 2 medication failures from the same class (decrease). Key barrier to use changes included time to results (decrease). 3 PGT were completed during the project. All patients met the protocol criteria for testing. None of these patients had medication failures documented using theEHR template; all of the patients did have documentation using each prescriber's preferred method. 2 of the 3 tests were uploaded to the EHR. The first test completed was not integrated, likely due to support staff becoming accustomed to the new workflow. 117 historical PGT were also integrated into the EHR.

CONCLUSIONS: While 16 to $20 \%$ of the population meets the criteria for MDD, available treatments achieve symptom remission only $40 \%$ of the time (Singh, 2014). Patients who do not achieve remission experience relapse more quickly and are more likely to develop chronic non-remitting MDD (Gaynes, 2016). While the PGT evidence base is still evolving, its use in clinical practice has the potential to improve depression treatment outcomes. This study highlighted continued barriers to PGT use in a practice setting, while implementing key interventions, including PGT use guidelines and EHR integration, to improve its systematic and appropriate use.

FUNDING ACKNOWLEDGEMENTS: No funding.

152

\section{Pimavanserin for the Treatment of Parkinson's Disease Psychosis: Number Needed to Treat, Number Needed to Harm, and Likelihood to Be Helped or Harmed}

Leslie Citrome, MD, MPH'; James Norton, $P h D^{2}$; Kathy Chi-Burris, $\mathrm{MPH}^{3}$; and George Demos, $\mathrm{MD}^{4}$

${ }^{1}$ Clinical Professor or Psychiatry and Behavioral Sciences, Department of Psychiatry \& Behavioral Sciences, New York Medical College, Valhalla, NY
${ }^{2}$ Sr. Director Medical Affairs, ACADIA

Pharmaceuticals, Inc. San Diego, CA

${ }^{3}$ Sr. Director Biostatistics, ACADIA Pharmaceuticals, Inc. San Diego, CA

${ }^{4}$ Executive Director Drug Safety and Pharmacovigilance, ACADIA Pharmaceuticals, Inc. San Diego, CA

ABSTRACT: Study Objective: Psychosis is common in Parkinson's disease (PD) and increases in both frequency and severity with disease duration. It is associated with increased morbidity/mortality, complicates management of motor symptoms and often leads to long-term care placement. Pimavanserin (PIM) is a highly selective serotonin 5-HT2A receptor antagonist/inverse agonist indicated for the treatment of hallucinations and delusions associated with PD psychosis (PDP). The study aim is to review theevidence-base for PIM for the treatment of PDP using the metrics of evidence-based medicine, namely number needed to treat (NNT), number needed to harm $(\mathrm{NNH})$, andlikelihood to be helped or harmed $(\mathrm{LHH})$, in order to better place this intervention into clinical perspective.

METHODS: NNT and NNH are measures of effect size and indicate how many patients would need to be treated with one agent instead of the comparator in order to encounter one additional outcome of interest. A useful medication is one with a low NNT and a high NNH when comparing it with another intervention; a low NNT and a high NNH would mean one is more likely to encounter a benefit than a harm. Categorical efficacy and tolerability data was extracted from the clinical trial databases of the double-blind placebo-controlled studies of PIM in persons with PDP. The studies were 6 weeks in duration and fixed dose with the exception of study ACP-103-006 which was 4-weeks in duration. NNT and NNH values were calculated with their respective $95 \%$ confidence intervals. Efficacy endpoints were defined based on 2 definitions: a) Scale for the Assessment ofPositive Symptoms in Parkinson's Disease (SAPS-PD) total score decrease $\geq 3$ points from baseline and b) Clinical Global Impressions-Improvement scale (CGI-I) score of 1 (very much improved) or 2 (much improved). Tolerability outcomes of clinical interest, occurring at any time in available studies were assessed, including discontinuation due toan adverse event (AE). Likelihood to be helped or harmed (LHH) was then calculated contrasting therapeutic response vs. discontinuation because of an $\mathrm{AE}$.

RESULTS: NNT values for PIM $34 \mathrm{mg} / \mathrm{d}$ vs. placebo for several definitions of clinical response are $<10$, and as robust as 4 , denoting that PIM is a potentially efficacious intervention. $\mathrm{NNH}$ values for tolerability outcomes for PIM $34 \mathrm{mg} / \mathrm{d}$ (as well as for doses that range from $8.5 \mathrm{mg} / \mathrm{d}$ to $51 \mathrm{mg} / \mathrm{d}$ ) are $>10$, and/or 